\title{
The role of virtual reality on outcomes in rehabilitation of Parkinson's disease: meta-analysis and systematic review in 1031 participants
}

\author{
Joseph Triegaardt ${ }^{1} \cdot$ Thang S. Han $^{1,2}$ (1) $\cdot$ Charif Sada ${ }^{1} \cdot$ Sapna Sharma ${ }^{1} \cdot$ Pankaj Sharma ${ }^{1,3}$ \\ Received: 12 August 2019 / Accepted: 6 November 2019 / Published online: 6 December 2019 \\ (C) The Author(s) 2019, corrected publication 2019
}

\begin{abstract}
Introduction Parkinson's disease (PD) is managed primarily by dopamine agonists and physiotherapy while virtual reality (VR) has emerged recently as a complementary method. The present study reviewed the effectiveness of VR in rehabilitation of patients with PD.

Methods Literature search up to June 2019 identified ten studies ( $n=343$ participants) suitable for meta-analysis and 27 studies ( $n=688$ participants) for systematic review. Standard mean difference (SMD) and 95\% confidence intervals (CI) were calculated using a random effects model.

Results In meta-analysis, compared with active rehabilitation intervention, VR training led to greater improvement of stride length, $\mathrm{SMD}=0.70(95 \% \mathrm{CI}=0.32-1.08, p=0.0003)$, and was as effective for gait speed, balance and coordination, cognitive function and mental health, quality of life and activities of daily living. Compared with passive rehabilitation intervention, VR had greater effects on balance: $\mathrm{SMD}=1.02(95 \% \mathrm{CI}=0.38-1.65, p=0.002)$. Results from single randomised controlled trials showed that VR training was better than passive rehabilitation intervention for improving gait speed SMD $=1.43(95 \% \mathrm{CI}=0.51-2.34, p=0.002)$, stride length $\mathrm{SMD}=1.27(95 \% \mathrm{CI}=0.38-$ $2.16, p=0.005)$ and activities of daily living $\mathrm{SMD}=0.96(95 \% \mathrm{CI}=0.02-1.89)$. Systematic review showed that VR training significantly $(p<0.05)$ improved motor function, balance and co-ordination, cognitive function and mental health, and quality of life and activities of daily living.

Conclusion VR used in rehabilitation for patients with PD improves a number of outcomes and may be considered for routine use in rehabilitation.
\end{abstract}

Keywords Cognitive function $\cdot$ Neurological disorders $\cdot$ Physical function $\cdot$ Quality of life

Electronic supplementary material The online version of this article (https://doi.org/10.1007/s10072-019-04144-3) contains supplementary material, which is available to authorized users.

Thang S. Han

thang.han@rhul.ac.uk

$\triangle$ Pankaj Sharma

pankaj.sharma@rhul.ac.uk

1 Institute of Cardiovascular Research Royal Holloway, University of London, Egham, Surrey TW20 0EX, UK

2 Department of Endocrinology, Ashford \& St Peter's NHS Foundation Trust, Chertsey, UK

3 Department of Clinical Neuroscience, Imperial College Healthcare NHS Foundation, London, UK

\section{Introduction}

Parkinson's disease (PD), a progressive neurological disorder, affects the initiation and execution of voluntary movements, leading to difficulty in performing basic daily activities of living and impaired quality of life. PD is associated with shorter life expectancy [1]. Approximately 145,000 people were living with PD in the UK in 2018 and it is expected to rise to 168,000 by 2025 [2]. Dopamine agonists have been the principal drugs for treating PD since 1975 [3] but have a number of adverse effects including dopamine dysregulation syndrome, occurring in $4 \%$ of patients on long-term treatment [4], gambling addiction, excessive spending and sexual hyperfunction [4].

Physical rehabilitation is an essential complementary component to drug therapy of PD while virtual reality (VR) has 
increasingly been additionally applied to rehabilitation of patients with neurological conditions [5]. This technology is a computer-generated environment in which the user can perceive, feel and interact in a manner that is similar to a physical place, achieved by combining stimulation over multiple sensory channels such as sight, sound and touch [6]. Because of its ability to simulate real-world situations and cognitive and motor tasks in a safe environment, completion of VR tasks is a rewarding form of therapy for patients with PD. Other beneficial gain from VR training is the immediate feedback through the augmented reality that it provides, altering the senses that the patient experiences [7]. However, VR is a relatively novel technology and not yet routinely used in clinical practice since there are only handful of randomised controlled trials have been performed in patients with PD. The present study conducted a meta-analysis and systematic review to examine the effectiveness of VR in patients with PD.

\section{Methods}

\section{Literature search}

We followed guidelines from the Cochrane and PRISMA recommendations on conducting a meta-analysis [8,9]. Literature search of MEDLINE and Google Scholar was performed up to June 2019 using the key terms: 'virtual reality' and 'Parkinson's disease'. No language or data filters were applied. The Boolean operators were used to combine search terms. Relevant studies were traced within their references.

\section{Selection criteria}

Studies examining either idiopathic or familial PD irrespective of age, sex, drug dosage and duration of PD were included. Inclusion criteria for meta-analysis required the study to be a randomised controlled trial. Control group may be active (patients receiving an alternative therapy treatment, e.g. physiotherapy) or passive (without alternative therapy). VR may be fully immersive where the user wears a headset to view the task or non-immersive where the user views the task on computer or television monitor. The majority of studies included in meta-analysis used non-immersive Nintendo ${ }^{\mathrm{TM}}$ Wii (Nintendo; Redmond, WA, USA).

\section{Outcome measures}

The outcomes for the comparative analysis were gait, stride length, balance, global motor function, activities of daily life, Parkinson's Disease Questionnaire 39 (PDQ39) and cognitive function. It should be borne in mind that the definition of outcomes may vary between studies.

\section{Statistical analysis}

Meta-analysis was performed using Review Manager v5.3 (Copenhagen: The Nordic Cochrane Centre, The Cochrane Collaboration, 2014). The effect size was assessed by standard mean difference (SMD) and 95\% confidence intervals (CI) using random effects model. Statistical significance threshold was accepted as $p<0.05$. Inter-study heterogeneity was assessed by $\mathrm{I}^{2}$ test with a value of $>50 \%$ being considered substantial heterogeneity. Risk of bias was assessed using Cochrane Collaboration's tool [10].

\section{Results}

Using the search terms 'virtual reality' and 'Parkinson's disease' identified 21,300 articles. After screening for duplications and selection criteria, ten papers met selection criteria for meta-analysis and 27 papers suitable for systematic review (Fig. 1).

\section{Meta-analysis}

Ten papers comprised a total of 343 participants of mean age ranging from 61.1 to 78.4 years and disease duration from 6 to 9.4 years. A variety of VR systems were used with Nintendo ${ }^{\mathrm{TM}}$ Wii being the most popular (in half of all studies). The remaining trials included balance boards and other commercially available systems such as GestureTek IREX videocapture system $^{\mathrm{TM}}$ (Toronto, ON, Canada). Duration of the trials varied from just over 4 weeks [11] to 12 weeks [12].

Except for one study [13], all trials employed a control group that was active which included conventional physiotherapy, balance and gait training. The control for the study by Lee et al consisted of neurodevelopmental treatment followed by functional electrical stimulation [13]. Two studies included both active and passive control groups $[14,15]$. Due to inter-study heterogeneity of some of the trials, not all outcomes could be used in meta-analysis.

\section{Virtual reality intervention versus active intervention control}

Four trials comprising 116 patients assessed stride length [12, 15-17]. Compared with active intervention, VR training led to greater improvement of stride length: $\mathrm{SMD}=0.70(95 \% \mathrm{CI}=$ 0.32 to $1.08, p=0.0003)$. There was no evidence of interstudy heterogeneity $\left(\mathrm{I}^{2}=0.0 \%, p=0.90\right)$ (Fig. 2).

Six trials $(n=209$ participants) assessed gait [12, 15-19]. Gait speed was similarly improved both by VR and by active intervention but there were no differences in improvement between these two methods: $\mathrm{SMD}=0.08(95 \% \mathrm{CI}=-0.27$ to $0.44, p=0.65)$. There was no inter-study heterogeneity $\left(\mathrm{I}^{2}=\right.$ $34 \%, p=0.18$ ) (Supplementary Fig. 1). 


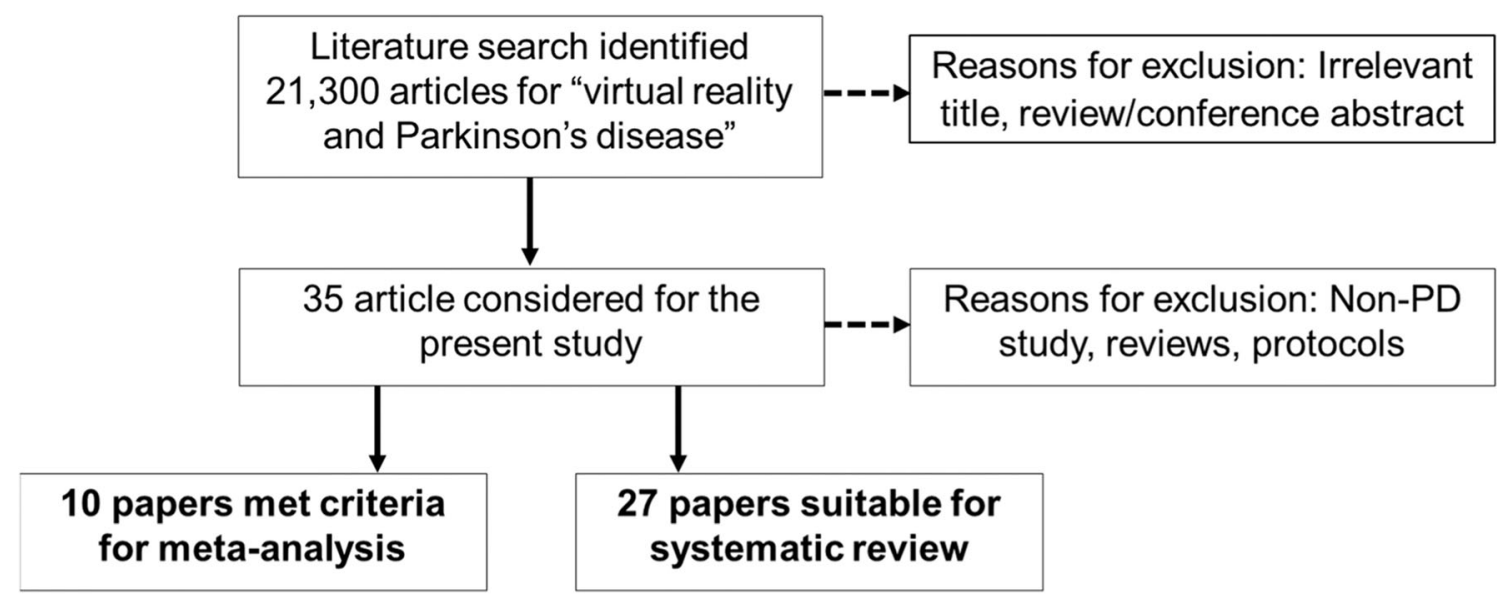

Fig. 1 Flowchart showing process of study selection in the present study

Five trials ( $n=166$ participants) examined balance using Berg Balance scale [16-20]. Improvement in balance did not differ between VR and active intervention: $\mathrm{SMD}=0.26$ $(95 \% \mathrm{CI}=-1.02$ and $0.62, p=0.37)$. There was evidence of inter-study heterogeneity $\left(\mathrm{I}^{2}=61 \%, p=0.04\right)$ (Supplementary Fig. 2).

Motor function assessed by unified Parkinson's disease rating scale (UPDRS) was studied in three papers comprising 75 participants $[11,16,18]$. No differences in motor function were observed between VR and active intervention: $\mathrm{SMD}=-$ $0.38(95 \% \mathrm{CI}=-1.45$ to $0.69, p=0.49)$. There was evidence of inter-study heterogeneity $\left(\mathrm{I}^{2}=80 \%, p=0.007\right)$ (Supplementary Fig. 3).

Quality of life was assessed in five studies comprising 176 patients [11, 15, 16, 18, 19] (four studies used PDQ39 [11, 15, $16,18]$ and one used shorthand form (PDQ8) [19]). No differences in quality of life were observed between VR and active intervention: $\mathrm{SMD}=0.20(95 \% \mathrm{CI}=-0.16$ to $0.57, p$ $=0.27)$. There was no inter-study heterogeneity $\left(\mathrm{I}^{2}=28 \%, p=\right.$ 0.23) (Supplementary Fig. 4).

\section{Virtual reality intervention versus passive intervention}

Two trials on balance in 44 subjects $[13,15]$ showed that VR was significantly better than passive intervention on balance: $\mathrm{SMD}=1.02(95 \% \mathrm{CI}=0.38$ to $1.65, p=0.002)$. There was no heterogeneity found between the studies (Supplementary Fig. 5).

\section{Single randomised controlled trials}

We found only a single study comparing VR and active intervention on activities of daily living and cognitive function comprising 32 subjects [20]. There were no differences in activities of daily living SMD $=-0.13(95 \% \mathrm{CI}=-0.82$ and $0.57, p=0.72$ ) or in cognitive function between VR and active intervention $\mathrm{SMD}=0.08(95 \% \mathrm{CI}=-0.61$ to $0.78, p=$ $0.81)$.

There were also single studies comparing VR against passive intervention. The single trial of 20 subjects by Lee et al. [13] showed VR led to greater improvement in activities of daily living than passive intervention $\mathrm{SMD}=0.96(95 \% \mathrm{CI}=$ 0.02 to $1.89, p=0.05$ ) and that by Liao et al [15] of 24 participants showed that VR had a greater improvement than passive intervention in gait speed $\mathrm{SMD}=1.43(95 \% \mathrm{CI}=0.51$ and 2.34, $p=0.002)$ and in stride length $\mathrm{SMD}=1.27(95 \% \mathrm{CI}$ $=0.38$ to $2.16, p=0.005$ ).

\section{Risk of bias of randomised controlled trials}

Figure 3 shows risk of bias assessments for the ten randomised controlled trials in the present study. Random sequence generation and allocation was examined and found only 2 papers $[13,17]$ to have an unclear risk of bias. The remaining trials reported methods of randomisation, therefore were considered to have low risk of bias. All the trials were blinded apart from two $[13,17]$. The trial by Pedreira et al. had an unclear risk of

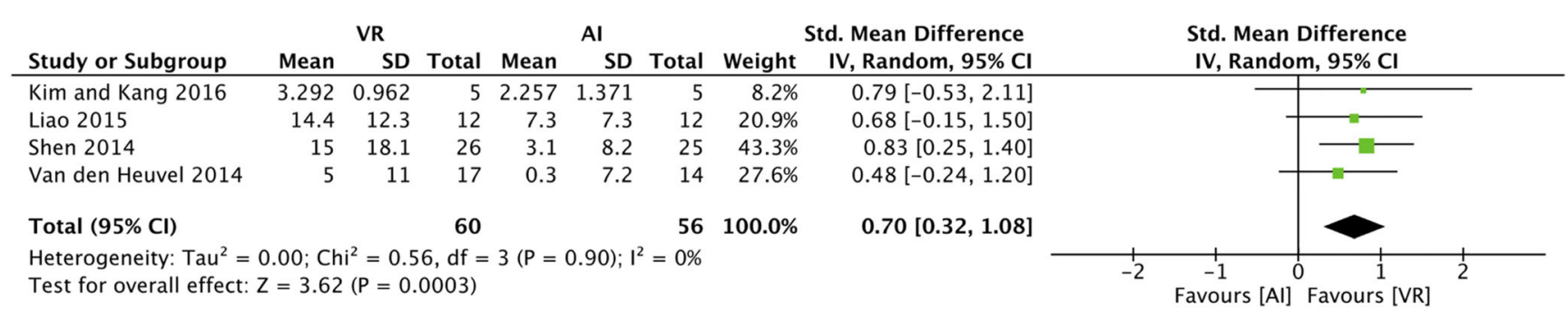

Fig. 2 Forest plot showing effects of VR training compared with active intervention on stride length 


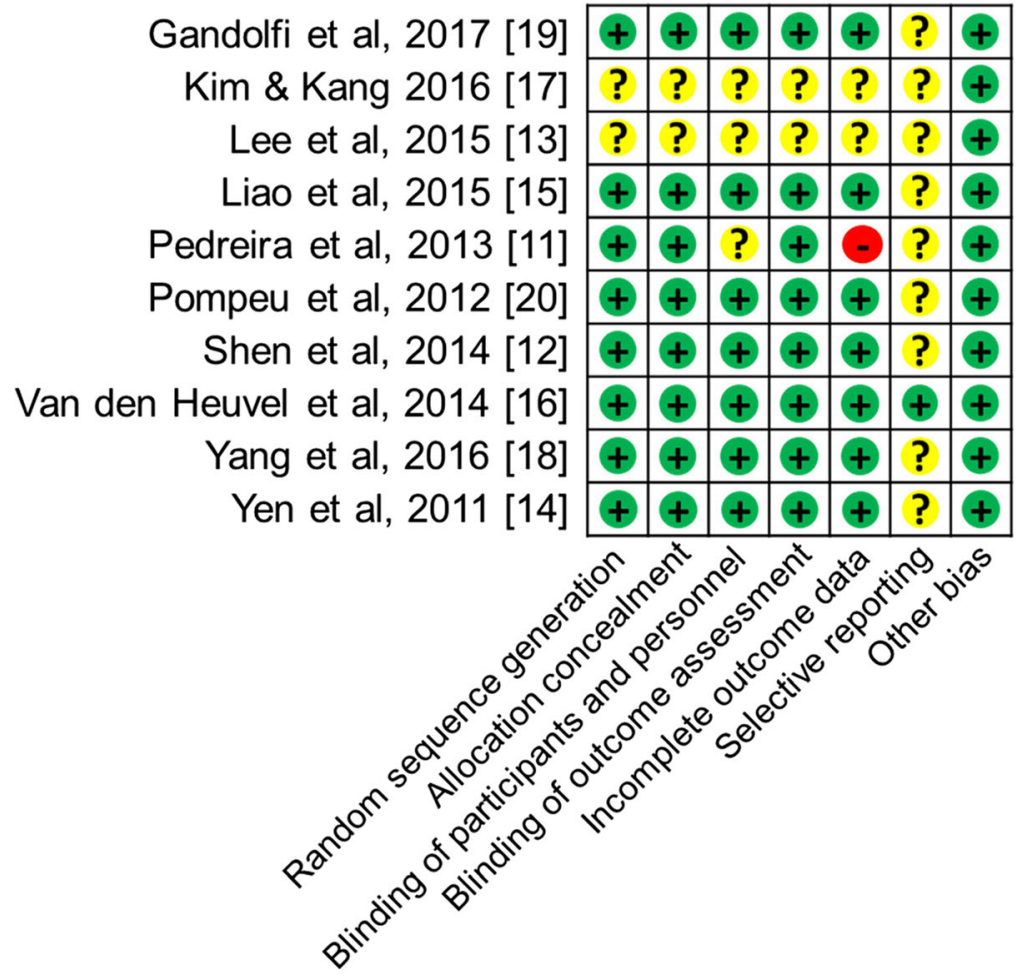

Key

† Low risk of bias

- High risk of bias

? Unclear risk of bias

Fig. 3 Risk of bias for meta-analysis studies

bias while the remaining trials, which were blinded, had low risk of bias [11]. Three studies had an unclear risk of incomplete outcome data bias $[13,15,17]$. The rest of the trials either had no drop-outs or they used intent to treat analysis to compensate for drop-outs [12, 14, 16, 18, 19] while the study by Pedreira et al. had a high risk of bias [11]. Selective reporting bias was unclear in most studies as protocol was not mentioned, only 1 paper reported the study protocol [16], therefore was considered to have a low risk of bias.

\section{Systematic review}

A total of 27 papers comprising 688 participants met selection criteria for the systematic review. Participant characteristics and study features are shown in Supplementary Table 1. The mean age ranged between 60.3 and 72 years and PD duration between 5.1 and 9.8 years. The majority papers examined the effect of VR on motor outcomes including balance, gait or activities of daily living while quality of life was assessed in 23 papers. Significant improvements in at least one of the outcomes by VR were reported in 18 papers. Nintendo ${ }^{\mathrm{TM}}$ Wii was the most popular VR system (11 studies) followed by Xbox Kinect ${ }^{\mathrm{TM}}$ (Microsoft Corporation, Redmond, WA, USA) (four studies) while two studies used custom made VR systems. The remaining ten studies used other commercially available VR systems such as Oculus Rift headmounted displays (Facebook Technologies, LLC, Menlo Park, CA, USA).
Studies often assessed the effects of VR on different outcomes. For clarity, outcomes were classified into groups of (1) motor function which includes gait and stride length, endurance, walking distance, Timed Up and Go, Sitting to Standing Test and tapping; (2) balance and co-ordination; (3) cognitive function and mental health including Geriatric Depression Scale and (4) quality of life and activities of daily living.

\section{Motor function}

VR training has been shown to improve Timed Up and Go a decrease of $1.9 \mathrm{~s}$ in Timed Up and Go test compared with a decrease of $1.2 \mathrm{~s}$ in control group ( $p=0.040$ ) [21]. Zettergren et al. found an improvement in Timed Up and Go by $34 \%$ as well as gait speed by $42 \%$ after VR training twice a week for 8 weeks [22]. Badarny et al. observed that $65 \%$ of patients improved either gait speed or stride length or both by more than $10 \%$ with VR training $(p=0.002)$ [23]. These findings are supported by a study by Palacios-Navarro et al. who observed that VR training significantly increased in gait speed ( $p=$ 0.002 ), reducing the completion time from 12 to $10 \mathrm{~s}$ [24]. de Melo et al. found that compared with conventional training, VR intervention led to faster gait speed $(p=0.031)$ and higher Borg score (a measure of physical fitness) $(p=0.005)$ [25], and similarly, Mirelman et al. showed gait speed increased in normal walking ( $p=0.006)$ and in obstacle negotiation $(0.001)$ and stride increased in normal walking $(p=0.043)$ and in obstacle negotiation $(p=0.019)$ [26]. Mirelman et al. 
also found that UPDRS was significantly $(p=0.020)$ increased with VR training [26]. VR training has also led to improvements in Sitting to Standing Test $(p=0.010), 10$ Metre Walk Test and Performance Orientated Mobility Assessment Test $(p=0.050)$ [21] and 6-Minute Walk Test ( $p$ $=0.043$ ) [17]. There was only one study on tapping frequency showing VR training had no significant effects in patients with PD [27]. A number of other studies found VR training led to improvement in gait speed [12, 15, 18, 20], stride length [15], Timed Up and Go [15, 18, 28] and UPDRS [20, 28]. Although Yang et al. did not find significant improvement in UPDRS [18].

\section{Balance and co-ordination}

Esculier et al. found that VR training in PD patients led to significant improvement in one leg stance duration $(p=$ 0.020 ), community balance and mobility score by 15 points compared with improvement in control group of 7.5 points ( $p$ $=0.001)$ [21]. VR training improved activities-specific balance confidence $(\mathrm{ABC})$ test by $6 \%(p=0.025)[12]$ but not balance assessed by Centre of Pressure Length score [29]. In contrast, Loureiro et al. showed VR training improved Borg scale $(p=0.046)$, Berg Balance Scale $(p=0.046)$ and functional reach to the left $(p=0.043)$ and right $(p=0.028)$ [30].

Balance has been shown to improve by VR training in a number of other studies [13, 18, 20,31]. VR training also improved unipedal stance test $(p=0.050)$ [20] and Balance Evaluation System Test score from 74.1 to 88.9 [32]. Severiano et al. found improvement in the Dizziness Handicap Inventory $(p=0.022)$ after VR intervention [31]. Gandolfi et al. investigated the effect of VR and Sensory Balance Integration Training on balance showing both methods led to significant ( $p=0.040)$ improvement of Berg Balance Scale scores: VR group improved by 3.74 and Sensory Balance Integration Training group by 4.21 points. Although there were no differences in satisfaction rates between study groups, the cost of VR rehabilitation was cheaper than that of Sensory Balance Integration Training group by $€ 5600$ [19].

Herz et al. found VR training led to significant increases in Purdue Pegboard Test score $(p=0.011)$, Timed Tap score ( $p=$ $0.003)$ and 9 hole peg test $(p=0.033)$ [28], while Ma et al. observed that VR training resulted in more force $(p=0.005)$ and quicker time $(p=0.005)$ to reach for stationery balls than the control [33]. A study by van den Heuvel et al. found VR training did not improve Functional Reach Test [16]. A study by Kim and Kang showed VR training resulted in significant greater increase than control in weight bearing distribution difference (with eyes open) $(p=0.038)$ and Berg Balance Scale $(p=0.043)$, improvement in mediolateral and anteroposterior sway length, ground reaction force and step length $(p=0.043)$ [17], while Liao et al. found improvement in Fall Efficacy Scale $(p<0.001)[15]$ and Yen et al. found improvement in sensory integration for postural control $(p<$ 0.001) [14].

\section{Cognitive function and mental health}

Pompeu et al. showed VR training significant improved Montreal cognitive assessment $(p=0.001)$ [20]. Cipresso et al. assessed Executive Function after VR training found that patients with $\mathrm{PD}$ with normal cognition were better than those with PD with mild cognitive impairment in performing Clock Drawing Test $(p=0.001)$, Phonological Fluency Test $(p=$ $0.001)$, semantic verbal fluency test $(p=0.001)$ and the Tower of London Test $(p=0.001)$ as well as strategies $(p=$ 0.001 ) [34]. There were no group differences in performing Virtual Multiple Errands Test or rule breaks. Mirelman et al. found VR training did not improve mistakes during Serial Subtraction Test $(p=0.160)$ [26].

Lee et al. found VR training led to a significant decrease in Beck Depression Inventory score $(p<0.050)[13]$ while Herz et al. found no improvement on Hamilton Depression Scale scores [28].

\section{Quality of life and activities of daily living}

VR training has been shown to significantly improve quality of life by several studies using PDQ39 [11, 15, 18, 20, 28] while Severiano et al. found significant improvement in quality of life assessed by Short Form-36 by tight rope $(p=0.045)$ and ski slalom games $(p=0.012)$ [31]. VR training also led to improvement in activities of daily living assessed by Modified Barthel Index $(p<0.050)$ [13] or by Nottingham Extended Activities of Daily Living Test score $(p=0.015)$ [28].

\section{Risk of bias in systematic review studies}

Figure 4 shows risk of bias for systematic review. For random sequence generation and allocation, 15 studies showed a high risk, two unknown risk, ten low risk of allocation bias. Blinding was split into blinding of participants and of outcome assessment; 18 studies showed a high risk, three unclear and six low risk of bias. Blinding of the examiners is arguably easier in studies that require high level of patient interaction; thus, more studies were found to have a low risk of bias for blinding of the examiners; 14 studies showed a high risk, 11 low risk and two unclear risk of bias. Incomplete outcome data examined the number of drop-outs and found six studies to have a high risk, three unclear risks and 18 low risk of bias for incomplete data. Selective reporting bias was based on the availability of study protocol; four studies had a low risk while 23 had an unknown bias. 


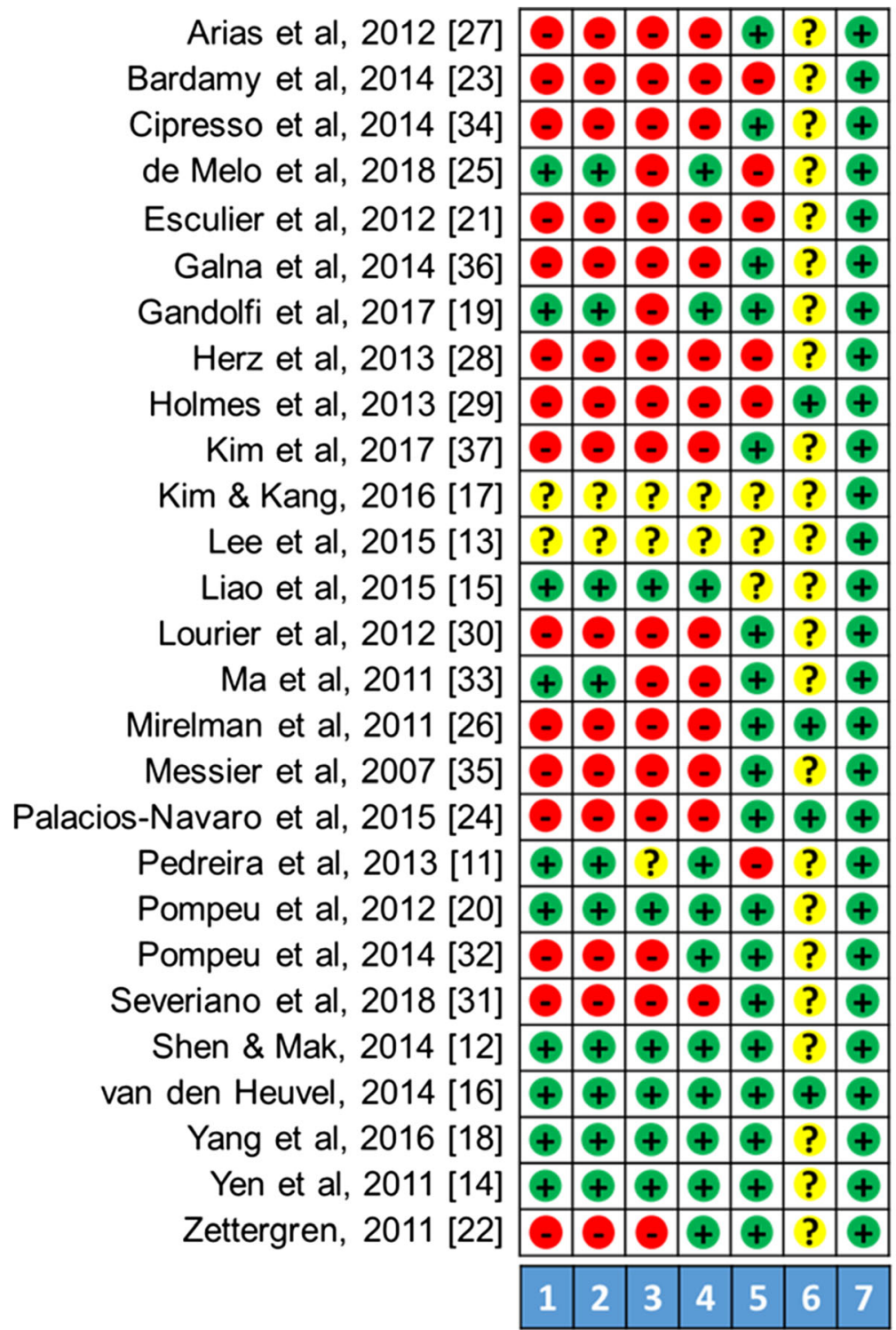

Key

† Low risk of bias

- High risk of bias

? Unclear risk of bias
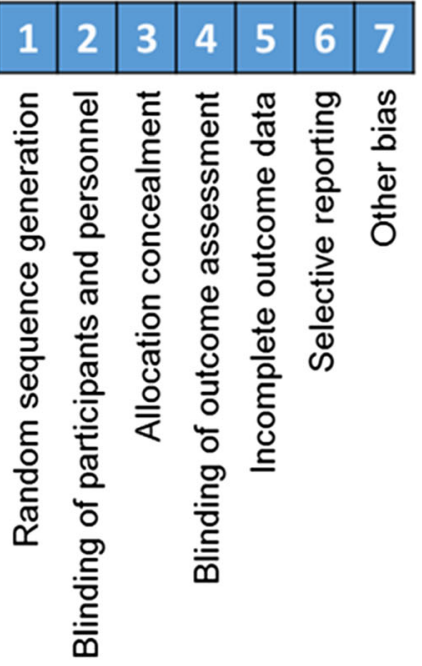

Fig. 4 Risk of bias for systemic review studies

\section{Discussion}

The present meta-analysis and systematic review of over a thousand participants found VR training improved a number of outcomes in patients with PD including motor functioning, balance and co-ordination, cognitive function and quality of life. VR is a relatively new technology applied to the field of clinical medical; therefore, there is a paucity of data on its effectiveness. Findings from our study of a relatively large number of subjects are valuable in providing scientific evidence to healthcare specialists when considering to integrate VR training into rehabilitation programme.

There are a number of benefits in using VR with or without conventional physiotherapy. The use of commercially available sources of VR has the potential to allow an increased number of patients to access care from the comfort of their own home, reducing the need to travel to access care. VR training is safe and interesting [7]. The lower cost is also attractive to healthcare organisations. A recent study has found that the cost of VR rehabilitation for 36 patients with PD over 7 weeks was $€ 23,299$ compared to $€ 28,890$ for 34 patients using balance training [19]. This saving of $€ 5,590$ is enormous if most of patients with PD undergo VR training.

Pompeu et al. [32] have demonstrated that patients' VR game performance increased over time (from first to last session) $(p<0.05)$ and found no adverse effects from playing the games. There were few studies showing poorer outcomes after VR training including that of Messier et al. who found patients with PD were worse at visuomotor learning compared to the age matched controls [35]. Galna et al. assessed game design and game feasibility found that patients preferred the games to be at a slow pace over narrative driven games. Patients tended 
to enjoy games with no negative effects. Most participants felt safe using the equipment and would prepare to use the system at home. Some patients had difficulty with stepping tasks and some of the objects within the game [36]. Kim et al. observed no significant adverse effects such as sickness or balance issues as a result of exposure to VR while levels of stress decreased and arousal increased [37].

The value of VR in rehabilitation of patients with neurological disorders has been recognised by a number of clinical specialists for about two decades [38-40]. The use of VR rehabilitation for other neurological conditions such as stroke has been shown to have similar beneficial effects to those used in patients with PD including physical or motor function [41-43], activities of daily living [42] and quality of life [43].

The exact mechanisms of how VR improves outcomes measure are not yet fully understood. VR has interested neuroscientists in the field of neuroergonomics research as a tool for neurorehabilitation [44] because of the human brain plasticity which enables it to adapt to environmental pressure, experiences and challenges, which could be replicated by VR, including patients with brain disorders including patients with PD or stroke [45-47].

\section{Limitations}

The major limitation lies in its small number of studies and participants, which is expected for a novel technology. We have therefore assessed comprehensively the risk of bias to address limitations of each individual paper. Most of the studies examined the effects of VR over a relatively short period; therefore, long-term outcomes are not known. The variety of different VR systems (nonimmersive and fully immersive) used by different trials may have affected the results since some system may confer greater advantage than others. VR technology is still evolving and higher quality and unified system may be available for specific use in rehabilitation of patients with different needs.

In conclusion, VR used in rehabilitation for patients with PD improves a number of outcomes and may be considered for routine use in rehabilitation.

Authors' contribution PS, CS and TSH reviewed the topic related literature and performed the study concept and analysis design. JT performed the data collection and wrote the first draft, analysed and interpreted the data. TSH and PS further edited the draft. PS, SS, and SC edited and TSH revised the manuscript. All authors checked, interpreted results and approved the final version.

\section{Compliance with ethical standards}

Conflict of interest The authors declare that they have no conflicts of interest.
Ethical approval This study was conducted in accordance with the 1964 Helsinki Declaration and its later amendments or comparable ethical standards.

Statement of human and animal rights This article does not contain any studies with animals performed by any of the authors.

Open Access This article is licensed under a Creative Commons Attribution 4.0 International License, which permits use, sharing, adaptation, distribution and reproduction in any medium or format, as long as you give appropriate credit to the original author(s) and the source, provide a link to the Creative Commons licence, and indicate if changes were made. The images or other third party material in this article are included in the article's Creative Commons licence, unless indicated otherwise in a credit line to the material. If material is not included in the article's Creative Commons licence and your intended use is not permitted by statutory regulation or exceeds the permitted use, you will need to obtain permission directly from the copyright holder. To view a copy of this licence, visit http://creativecommons.org/licenses/by/4.0/.

\section{References}

1. Chaudhuri KR, Healy DG, Schapira AH (2006) Non-motor symptoms of Parkinson's disease: diagnosis and management. Lancet Neurol 5:235-245

2. Parkinson's UK. The incidence and prevalence of Parkinson's in the UK. Results from the Clinical Practice Research Datalink Summary report. https://www.parkinsons.org.uk/sites/default/files/2018-01/ CS2960\%20Incidence $\% 20$ and $\% 20$ prevalence $\% 20$ report $\%$ 20branding\%20summary\%20report.pdf [Access June 2019]

3. Tolosa E, Martí MJ, Valldeoriola F, Molinuevo JL (1998) History of levodopa and dopamine agonists in Parkinson's disease treatment. Neurology 50(6 Suppl 6):S2-S10

4. O'sullivan SS, Evans AH, Lees AJ (2009) Dopamine dysregulation syndrome. CNS Drugs 23:157-170

5. Viñas-Diz S, Sobrido-Prieto M (2016) Virtual reality for therapeutic purposes in stroke: a systematic review. Neurología (English Edition) 31:255-277

6. Parsons T, Gaggioli A, Riva G (2017) Virtual reality for research in social neuroscience. Brain Sci 7:42

7. Sveistrup H (2004) Motor rehabilitation using virtual reality. J Neuroeng Rehabil 1:10

8. Van Tulder M, Furlan A, Bombardier C, Bouter L, Editorial Board of the Cochrane Collaboration Back Review Group (2003) Updated method guidelines for systematic reviews in the Cochrane Collaboration Back Review Group. Spine 28:1290-1299

9. Moher D, Liberati A, Tetzlaff J, Altman DG (2009) Preferred reporting items for systematic reviews and meta-analyses: the PRISMA statement. Ann Intern Med 151:264-269

10. Higgins JP, Altman DG, Gøtzsche PC, Jüni P, Moher D, Oxman AD, Savović J, Schulz KF, Weeks L, Sterne JA (2011) The Cochrane Collaboration's tool for assessing risk of bias in randomised trials. BMJ 343:d5928

11. Pedreira G, Prazeres A, Cruz D, Gomes I, Monteiro L, Melo A (2013) Virtual games and quality of life in Parkinson's disease: a randomised controlled trial. Advances in Parkinson's Disease 2:97101

12. Shen X, Mak MK (2014) Balance and gait training with augmented feedback improves balance confidence in people with Parkinson's disease: a randomized controlled trial. Neurorehabil Neural Repair 28:524-535 
13. Lee NY, Lee DK, Song HS (2015) Effect of virtual reality dance exercise on the balance, activities of daily living, and depressive disorder status of Parkinson's disease patients. J Phys Ther Sci 27: 145-147

14. Yen CY, Lin KH, Hu MH, Wu RM, Lu TW, Lin CH (2011) Effects of virtual reality-augmented balance training on sensory organization and attentional demand for postural control in people with Parkinson disease: a randomized controlled trial. Phys Ther 91: 862-874

15. Liao YY, Yang YR, Cheng SJ, Wu YR, Fuh JL, Wang RY (2015) Virtual reality-based training to improve obstacle-crossing performance and dynamic balance in patients with Parkinson's disease. Neurorehabil Neural Repair 29:658-667

16. van den Heuvel MR, Kwakkel G, Beek PJ, Berendse HW, Daffertshofer A, van Wegen EE (2014) Effects of augmented visual feedback during balance training in Parkinson's disease: a pilot randomized clinical trial. Parkinsonism Relat Disord 20:13521358

17. Kim Y, Kang S (2016) Effects of virtual reality-based exercise on balance, gait, and falls efficacy in patients with Parkinson's disease: a pilot study. Journal of the Korean Society of Integrative Medicine 4:1-11

18. Yang WC, Wang HK, Wu RM, Lo CS, Lin KH (2016) Home-based virtual reality balance training and conventional balance training in Parkinson's disease: a randomized controlled trial. J Formos Med Assoc 115:734-743

19. Gandolfi M, Geroin C, Dimitrova E, Boldrini P, Waldner A, Bonadiman S, Picelli A, Regazzo S, Stirbu E, Primon D, Bosello C (2017) Virtual reality telerehabilitation for postural instability in Parkinson's disease: a multicenter, single-blind, randomized, controlled trial. Biomed Res Int 2017 Article ID 7962826

20. Pompeu JE, Mendes FA, Silva KG, Lobo AM, Oliveira Tde P, Zomignani AP, Piemonte ME (2012) Effect of Nintendo Wii ${ }^{\mathrm{TM}}$ based motor and cognitive training on activities of daily living in patients with Parkinson's disease: a randomised clinical trial. Physiotherapy 98:196-204

21. Esculier JF, Vaudrin J, Bériault P, Gagnon K, Tremblay LE (2012) Home-based balance training programme using Wii Fit with balance board for Parkinson's disease: a pilot study. J Rehabil Med 44: $144-150$

22. Zettergren K, Franca J, Antunes M, Lavallee C (2011) The effects of Nintendo Wii Fit training on gait speed, balance, functional mobility and depression in one person with Parkinson's disease. Applied Innovations \& Technologies 5:38-44

23. Badarny S, Aharon-Peretz J, Susel Z, Habib G, Baram Y (2014) Virtual reality feedback cues for improvement of gait in patients with Parkinson's disease. Tremor Other Hyperkinet Mov 4:225

24. Palacios-Navarro G, García-Magariño I, Ramos-Lorente P (2015) A Kinect-based system for lower limb rehabilitation in Parkinson's disease patients: a pilot study. J Med Syst 39:103

25. de Melo GE, Kleiner AF, Lopes JB, Dumont AJ, Lazzari RD, Galli M, Oliveira CS (2018) Effect of virtual reality training on walking distance and physical fitness in individuals with Parkinson's disease. NeuroRehabilitation 42:473-480

26. Mirelman A, Maidan I, Herman T, Deutsch JE, Giladi N, Hausdorff JM (2011) Virtual reality for gait training: can it induce motor learning to enhance complex walking and reduce fall risk in patients with Parkinson's disease? J Gerontol A Biol Sci Med Sci 66:234 240

27. Arias P, Robles-García V, Sanmartín G, Flores J, Cudeiro J (2012) Virtual reality as a tool for evaluation of repetitive rhythmic movements in the elderly and Parkinson's disease patients. PLoS One 7: e30021

28. Herz NB, Mehta SH, Sethi KD, Jackson P, Hall P, Morgan JC (2013) Nintendo Wii rehabilitation ("Wii-hab") provides benefits in Parkinson's disease. Parkinsonism Relat Disord 19:1039-1042
29. Holmes JD, Gu ML, Johnson AM, Jenkins ME (2013) The effects of a home-based virtual reality rehabilitation program on balance among individuals with Parkinson's disease. Physical \& Occupational Therapy in Geriatrics 31:241-253

30. Loureiro AP, Ribas CG, Zotz TG, Chen R, Ribas F (2012) Feasibility of virtual therapy in rehabilitation of Parkinson's disease patients: pilot study. Fisioter Mov Curitiba 25:659-666

31. Severiano MI, Zeigelboim BS, Teive HA, Santos GJ, Fonseca VR (2018) Effect of virtual reality in Parkinson's disease: a prospective observational study. VArq Neuropsiquiatr 76:78-84

32. Pompeu JE, Arduini LA, Botelho AR, Fonseca MB, Pompeu SA, Torriani-Pasin C, Deutsch JE (2014) Feasibility, safety and outcomes of playing Kinect Adventures! ${ }^{\mathrm{TM}}$ for people with Parkinson's disease: a pilot study. Physiotherapy 100:162-168

33. Ma HI, Hwang WJ, Fang JJ, Kuo JK, Wang CY, Leong IF, Wang TY (2011) Effects of virtual reality training on functional reaching movements in people with Parkinson's disease: a randomized controlled pilot trial. Clin Rehabil 25:892-902

34. Cipresso P, Albani G, Serino S, Pedroli E, Pallavicini F, Mauro A, Riva G (2014) Virtual multiple errands test (VMET): a virtual reality-based tool to detect early executive functions deficit in Parkinson's disease. Front Behav Neurosci 8:405

35. Messier J, Adamovich S, Jack D, Hening W, Sage J, Poizner H (2007) Visuomotor learning in immersive 3D virtual reality in Parkinson's disease and in aging. Exp Brain Res 179:457-574

36. Galna B, Jackson D, Schofield G, McNaney R, Webster M, Barry G, Mhiripiri D, Balaam M, Olivier P, Rochester L (2014) Retraining function in people with Parkinson's disease using the Microsoft Kinect: game design and pilot testing. J Neuroeng Rehabil 11:60

37. Kim A, Darakjian N, Finley JM (2017) Walking in fully immersive virtual environments: an evaluation of potential adverse effects in older adults and individuals with Parkinson's disease. J Neuroeng Rehabil 14:16

38. Jack D, Boian R, Merians AS, Tremaine M, Burdea GC, Adamovich SV, Recce M, Poizner H (2001) Virtual realityenhanced stroke rehabilitation. IEEE Trans Neural Syst Rehabil Eng 9:308-318

39. Rose FD, Brooks BM, Rizzo AA (2005) Virtual reality in brain damage rehabilitation. CyberPsychol Behav 8:241-262

40. Holden MK (2005) Virtual environments for motor rehabilitation. CyberPsychol Behav 8:187-211

41. Saposnik G, Levin M, Stroke Outcome Research Canada (SORCan) Working Group (2011) Virtual reality in stroke rehabilitation: a meta-analysis and implications for clinicians. Stroke 42: $1380-1386$

42. Laver K, George S, Thomas S, Deutsch JE, Crotty M (2012) Cochrane review: virtual reality for stroke rehabilitation. Eur J Phys Rehabil Med 48:523-530

43. Shin JH, Park SB, Jang SH (2015) Effects of game-based virtual reality on health-related quality of life in chronic stroke patients: a randomized, controlled study. Comput Biol Med 63:92-98

44. Kearney JK, Rizzo M, Severson J (2007) Virtual reality and neuroergonomics. Neuroergonomics:253-274

45. Johansson BB. Current trends in stroke rehabilitation. A review with focus on brain plasticity. Acta Neurol Scand 123:147-59.

46. Särkämö T, Altenmüller E, Rodríguez-Fornells A, Peretz I (2016) Music, brain, and rehabilitation: emerging therapeutic applications and potential neural mechanisms. Front Hum Neurosci 10:103

47. Li S (2017) Spasticity, motor recovery, and neural plasticity after stroke. Front Neurol 8:120

Publisher's note Springer Nature remains neutral with regard to jurisdictional claims in published maps and institutional affiliations. 